\title{
Free induction decay of a superposition stored in a quantum dot
}

\author{
A. J. Bennett, ${ }^{1}$ M. A. Pooley ${ }^{1,2}$ R. M. Stevenson,${ }^{1}$ I. Farrer,${ }^{2}$ D. A. Ritchie,${ }^{2}$ and A. J. Shields ${ }^{1}$ \\ ${ }^{1}$ Toshiba Research Europe Limited, Cambridge Research Laboratory, \\ 208 Science Park, Milton Road, Cambridge, CB4 OGZ, U. K. \\ ${ }^{2}$ Cavendish Laboratory, Cambridge University, \\ J. J. Thomson Avenue, Cambridge, CB3 OHE, U. K.
}

(Dated: April 8, 2018)

\begin{abstract}
We study the free evolution of a superposition initialized with high fidelity in the neutral-exciton state of a quantum dot. Read-out of the state at later times is achieved by polarized photon detection, averaged over a large number of cycles. By controlling the fine-structure splitting (FSS) of the dot with a DC electric field we show a reduction in the degree of polarization of the signal when the splitting is minimized. In analogy with the "free induction decay" observed in nuclear magnetic resonance, we attribute this to hyperfine interactions with nuclei in the semiconductor. We numerically model this effect and find good agreement with experimental studies. Our findings have implications for storage of superpositions in solid state systems, and for entangled photon pair emission protocols that require a small value of FSS.
\end{abstract}

PACS numbers:

Quantum effects are often masked by interactions with the environment. A well-known example is found in magnetic resonance spectroscopy. Typically, a radiofrequency pulse is used to prepare the nuclear spin states, which then precess around the applied magnetic field [1]. The signal obtained from simultaneously measuring the projection of all spins along some direction perpendicular to the field displays oscillations which appear to fall away with time in a process known as "free induction decay" (FID). In part this FID is due to the intrinsic decoherence of the spins, a so called $T_{2}$ process. But in the solid state this is often masked by a faster decay in the signal which arises from variations in the field, susceptibility and local environment of the nuclei, which consequently precess at different rates.

Quantum science can now routinely probe single solid state quantum systems, such as the spin of electrons trapped at color centers in diamond [2] (which has a weak phonon interaction) or single spins in silicon [3] (in which the host lattice has nuclear spin zero). On the other hand III-V semiconductors have both phonon and nuclear interactions to contend with, but are nonetheless interesting for their potential scalability and miniaturization, particularly as sources of non-classical light [4, 5].

One of the most studied systems are single InGaAs/GaAs quantum dots as they have optically active states with well-understood selection rules, allowing spinphoton conversion [6-10] and optical control [11, 12]. Interactions of these states with the nuclear spins in the quantum dot has lead to a wealth of new physics, such as "dragging" the energy of a transition as it follows a resonant laser [13, 14] and nuclear-spin switching [15]. Most of the literature on the effect of nuclear spins in a quantum dot has been concerned with the spin eigenstates of the charge-exciton transition [7, 12 14, 16, 17] or in some cases the neutral exciton transition in applied external magnetic field [14, 15, 18]. Through this work it has been shown that the hole has a hyperfine interaction which is an order of magnitude weaker than that of the electron [17, 19]. In contrast, our work is focussed on the behavior of superpositions stored in the neutral-exciton at zero external magnetic field. Study of this system is motivated by its ability to act as a a photon-exciton interface which will find applications in storage and manipulation of photons [6, 8, 10] and also in the its central role in the emission of entangled photon pairs from the neutral-cascade [5]. We show that the fluctuating magnetic field of nuclei overlapping with the wavefunction of the exciton have a pronounced effect on the time variation of the stored superposition. In analogy with NMR where a large array of spins are measured simultaneously, our experiment probes a single quantum system repeatedly over many fluctuations, averaging the signal to observe a similar FID. Controlling the energy splitting of the exciton eigenstates we are able to study the FID of stored superpositions in the dot. We find a faster FID is observed at small values of splitting, a result of the randomly varying nuclear field. Intriguingly, we also find that when the splitting is increased the FID timescale tends to a finite value that is similar for all dots.

We begin by discussing our experiments. A single InGaAs/GaAs quantum dot is excited one LO-photon energy (32 meV) above the exciton transition using a mode-locked laser running at $80 \mathrm{MHz}$. With this excitation scheme there is no phase-coherence between the laser and the initialized state and the laser can be spectrally filtered from the signal. However a coherent superposition between the populations of the eigenstates is created, mapping the polarization of the laser directly onto the Bloch-sphere of the solid-state exciton spin [6, 10]. When the exciton later radiatively decays the emitted light is passed to a fast silicon avalanche photodiode (response time below $100 \mathrm{ps}$ ) and polarized time-resolved data is acquired using counting electronics synchronized to the driving laser. Traces are acquired in tens of seconds with 
measurements parallel and orthogonal to the polarization of the laser. This is carried out in all three measurement bases defined by the linear eigenstates (LE), the linear superposition (LS) and circular superposition (CS). Each pair is used to calculate the "degree of polarization" (the difference in the traces divided by the sum) for that basis, such as the data shown in Figure 1f. The degree of polarization displays a noise which increases with time, $t$, as the signal falls with a radiative lifetime of $1.3 \mathrm{~ns}$. However, a good fit to the first $4 \mathrm{~ns}$ of data can be obtained using a least squares-fitting algorithm, to a function of the form $\propto \sin \left(|s|\left(t-t_{0}\right) / \hbar\right) \cdot \exp \left(-t / \tau_{F I D}\right)$, where the term in $\tau_{F I D}$ approximates an exponential decay to the FID signal and $t_{0}$ describes the phase of the oscillations. $s$ is the fitted fine-structure splitting (FSS) between the two eigenstates. In the absence of a magnetic field, anisotropy in the shape and strain of the dot, plus a contribution from crystal asymmetry leads to a finite splitting between linear eigenstates, $\left|s_{\text {linear }}\right|$, aligned with the crystal axes [5].

Our samples contain dots located in the center of a $p-i-n$ diode with $A l_{0.75} G a_{0.25} A s$ barriers on either side, which allow vertical electric fields to change the FSS [20]. We observe a minimum value of FSS, $\left|s_{0}\right|$, which varies from dot to dot. Figure 1 shows experimental data from the exciton state of one quantum dot with $\left|s_{0}\right|<0.4 \mu \mathrm{eV}$ at $-153 \mathrm{kV} / \mathrm{cm}$. Away from the minimum value of FSS the LE are horizontally and vertically linearly polarized in the laboratory frame, as is well known for dots of this type. This is evidenced in Figure 1f which shows that excitation with light aligned with this LE retains a high degree of polarization over the measurement. When the exciton is excited in a superposition the finite value of FSS leads to quantum beats in the intensity measured in a polarized measurement with a period of $|s| / \hbar[6,10,21]$. In the example shown in Figure 1f $|s|=3.7 \mu \mathrm{eV}$, and the degree of polarization decays with a lifetime of $3.0 \mathrm{~ns}$. The envelope of the degree of polarization for the CS and LS bases appear to degrade at a greater rate than the signal for the linear eigenstate.

When the FSS is reduced to the minimum value, $\left|s_{0}\right|$, the eigenstates of the exciton are rotated to be diagonal and anti-diagonal in the laboratory frame [20] (Figure [1). Again, the degree of polarization from the LE measurement decays at a rather slow rate. However, in the LS and CS bases we expect to see an absence of oscillations and a similarly slow decay rate, but instead Figure $1 \mathrm{~g}$ shows the degrees of polarization both fall to zero with decay time of $0.5 \mathrm{~ns}$. It appears the state has decohered, but as we shall show this is in fact a consequence of the hyperfine interactions with the nuclei in the sample.

The effect of a magnetic field on the eigenstates of the exciton has been well studied in other publications 22 24] and the known behavior is plotted in Figure [1d-e. For an externally applied vertical magnetic field (Faraday geometry) there is a splitting introduced in the circular basis of $s_{\text {circ }}=g_{X} \mu_{B} B$, where $g_{X}$ is the exciton g-factor (measured to be 3.0), $\mu_{B}$ the Bohr magneton
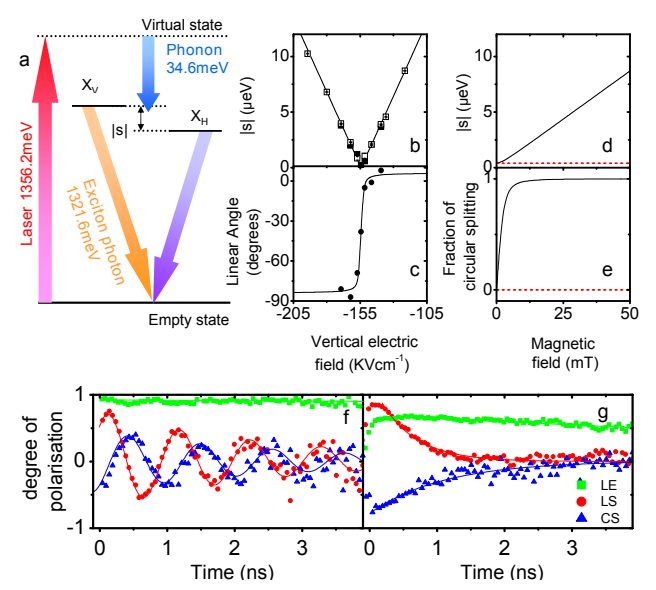

FIG. 1: (a) Level diagram for the phonon-assisted excitation of the neutral exciton states. Fine structure splitting (b) and eigenstate orientation (relative to the laboratory frame) measured in the spectral domain (filled data points) and temporal domain (open data points) for a dot with a minimum splitting $s_{0} \quad 0.4 \mu \mathrm{eV}$ as a function of vertical electric field. A fit [20] is included as a solid line. (d) Illustrates the magnitude of the total FSS at $s_{0}$ as a function of vertical (black) and in-plane (dashed) magnetic field used in the model. (e) shows the resulting fraction of the total FSS due to splitting in the circular basis as a function of magnetic field. (f) Time-resolved degree of polarization for excitation and detection along the linear eigenstate direction (LE, green), for the linear superposition direction (LS, red) and circular superposition direction (CS, blue) when the dot has a splitting of $3.7 \mu \mathrm{eV}$ at $-132 \mathrm{kV} / \mathrm{cm}$. (g) the same measurement when the splitting is minimal at $-153 \mathrm{kV} / \mathrm{cm}$.

and $B$ the applied field. This circular-basis splitting must be added in quadrature to find the total splitting,

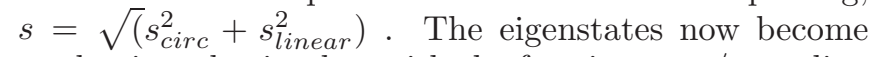
predominantly circular, with the fraction $s_{\text {circ }} / s$ tending to unity as shown in 1 . In contrast when the magnetic field is applied in the plane of the sample (Voigt geometry) the splitting increases only marginally according to $s=s_{\text {linear }}+\kappa B^{2}$ where $\kappa$ is of the order of $1-2 \mu \mathrm{eVT} T^{-2}$ [5, 23, 24]. For fields of up to $50 \mathrm{mT}$ this results in change in fine-structure splitting too small to measure.

Figure 2a shows the path of a superposition on the Bloch sphere for a pair of states with linear-basis splitting. The state precesses around the linear eigenstate (LE) axis and polarized measurements determine the averaged projection of the state along that particular axis as a function of time. We then assume that there is variable nuclear field which has some normal distribution in its magnitude and random orientation. We consider only the projection of the field $B_{N i}$ at some time along the vertical direction, leading to FSS in the circular basis $s_{\text {circ }}=g_{X} \mu_{B} B_{N i} \cos \left(\phi_{N i}\right)$, where $\phi_{N i}$ the angle between the field and the growth direction [22]. We approximate the nuclear spin interaction as an effective magnetic field and effective g-factor. However, to maintain generality we do not specifically assign the electron and hole contri- 
butions to the effective g-factor. The magnitude of the FSS is now $\left.\left|S_{N i}\right|=\sqrt{(} s_{\text {linear }}^{2}+\left(g_{X} \mu_{B} B_{N i} \cos \left(\phi_{N i}\right)\right)^{2}\right)$ [25]. The eigenstates of the exciton are rotated by an angle $\theta=\tan ^{-1}\left(\frac{g_{X} \mu_{B} B_{N i} \cos \left(\phi_{N i}\right)}{s_{\text {linear }}}\right)$. The state initialized then rotates around this new eigenstate at a rate of $S_{N i} / \hbar$, as shown in Figure $2 \mathrm{~b}$. To calculate the result of a measurement along the LS, LE and CS directions we extract the projection of this path along that direction.

$$
\begin{gathered}
V_{L S} \propto \cos \left(\left|S_{N i}\right| t / \hbar\right) \cdot e^{-t / \tau_{H V}} \\
V_{L E} \propto \sin \left(\left|S_{N i}\right| t / \hbar\right) \cdot \sin \left(\phi_{N i}\right) \cdot e^{-t / \tau_{H V}} \\
V_{C S} \propto-\sin \left(\left|S_{N i}\right| t / \hbar\right) \cdot \cos \left(\phi_{N i}\right) \cdot e^{-t / \tau_{H V}}
\end{gathered}
$$

In these equations we have also included an extra exponential decoherence term, with a timescale $\tau_{H V}$, the origin of which shall be discussed later.

In practice fluctuations in the nuclear field occur over a time scale of milliseconds [16, 22] which ensures that over a measurement many trajectories over the Bloch sphere are sampled. To calculate the effect this has we carry out Monte-Carlo simulations for 20,000 values of field magnitude and orientation denoted $i$. The results in Figure 2 (d) show the timescale of the FID due only to the hyperfine interaction (excluding the effect of $\tau_{H V}$ ) for different values of the initial linear splitting $\left|s_{\text {linear }}\right|$, as a function of the magnitude of the nuclear field fluctuations, where the different curves refer to varying Gaussian width of the distribution, in units of $g_{X} \mu_{B} B_{N i}$.

From this model we can infer a few interesting facts. (1) The nuclear field acts only to increase the total FSS, $\left|S_{N i}\right|$. Thus a temporal measurement of the FSS averaged over many $i$ should always lead to a marginally greater value than determined from a spectral measurement in the linear bases. Note that an averaging over $i$ leads to a circular splitting much less than $\left\langle g_{X} \mu_{B}\left|B_{N i}\right|\right\rangle$ as the field orientation is random. In turn this leads to an even smaller increase in the total splitting because the linear and circular splitting are added in quadrature. In practice, the error on the spectral measurement of FSS is greater than $0.3 \mu \mathrm{eV} \mathrm{[20]}$ so this difference between the temporal $|s|$ and spectral measurement of $\left|s_{\text {linear }}\right|$ cannot be observed (Figure 10). (2) From Figure 2c it can be seen that measurements of the free-induction decay will be different for measurements along the LS and CS axes. Consider for example the creation of a linear superposition, which is then measured along the linear superposition axis: in this case the measurement probes only the fluctuation in the magnitude of $S_{N i}$ but not the orientation of the eigenstates. In contrast, measurements projected along the CS axis are sensitive to both $B_{N i}$ and $\phi_{N i}$ and thus decay at a different rate. (3) Even when the excitation or measurement of the exciton is made in the time-averaged eigenstate, some weak oscillations will be observed as the state fluctuates into the circular basis.
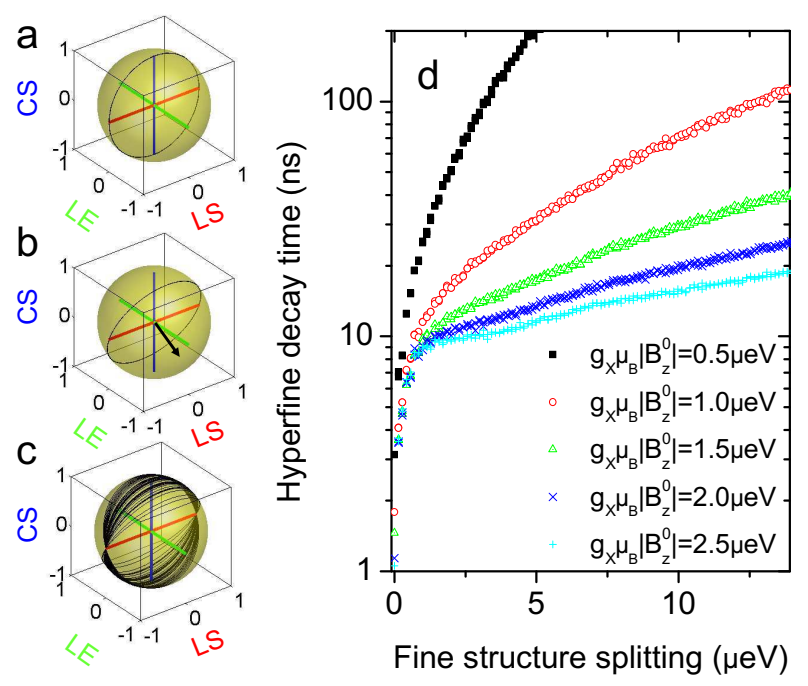

FIG. 2: (a) Bloch sphere for a qubit stored in the quantum dot with linear-basis splitting only. The state precesses around the Linear Eigenstate (LE) axis shown in green. (b) when a finite magnetic field is present the eigenstate is slightly shifted and the splitting is increased to $\left|S_{N i}\right|$. The superposition now evolves around the axis at a slightly increased rate $\left|S_{N i}\right| / \hbar$, and at a different angle. (c) when the distribution of fields and angles that can occur is considered it can be seen there are many possible paths. Performing Monte-Carlo simulations for 20,000 values of field, we can see the Free-induction decay of the degree of polarization due to this effect varies as shown in (d). Calculations were performed for different values of the initial linear splitting $\left|s_{\text {linear }}\right|$, and gaussian-widths to the magnitude of field fluctuations, in units of $g_{X} \mu_{B} B_{N i}$.

This is observed in our data, for example in the measurement of the LE shown in Figure 1f. (4) Finally, our model suggests that in the case where read out of the quantum trajectory can be made faster than the nuclear fluctuations this effect will be absent.

We have measured the rate of FID for a number of dots, as the FSS is varied (Figure 3). In all cases excitation with a low power density was used to ensure spin-pumping of the nuclear field was not occurring [15]. What we observe is that in the dot with the lowest $\left|s_{0}\right|$ (Figure 10 we see a rapid FID when the FSS is small (Figure 31 a). The variation in the FID time as a function of field (and FSS) is symmetric, suggesting is it an effect dominated by the absolute value of the FSS, not by the electric field. Measurements shown in Figure 3 on a dot where $\left|s_{0}\right|=2.5 \mu \mathrm{eV}$ show an accordingly greater FID time at the minimum FSS.

In Figure 3 the solid line represents the FID time extracted from our simulation for a Gaussian-width to the distribution nuclear fields of $g_{X} \mu_{B}\left|B_{N}\right|=2 \mu \mathrm{eV}$ and $\tau_{H V}$ $=3.0 \mathrm{~ns}$. Clearly, near $\left|s_{0}\right|$ the FID is dominated by the nuclear fluctuations, suggesting the width of the fluctuations in $\left|B_{N}\right|$ are of order $12 \mathrm{mT}$, and that several $10^{3}$ nuclei are interacting with the exciton. At larger values of splitting we see a decay determined by $\tau_{H V}$, which 
describes the characteristic rate at which the $H$ and $V$ components of the superposition lose relative phase ( the "cross dephasing time" [27], equivalent to the $T_{2}^{*}$ in NMR.

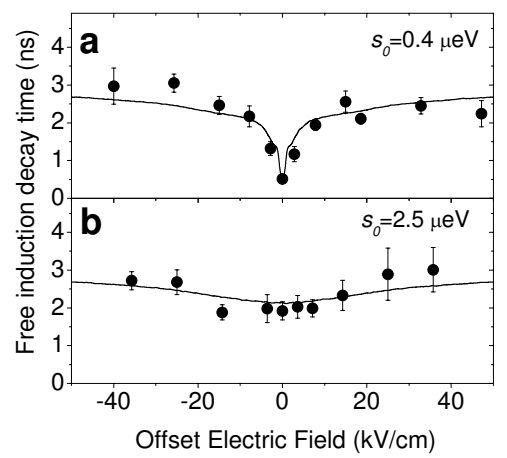

FIG. 3: Free induction decay time of a linear superposition stored in the exciton state of a single quantum dot. a For a dot with a minimum value of FSS of $0.4 \mu \mathrm{eV}$. b For a dot with a minimum value of FSS of $2.5 \mu \mathrm{eV}$

We also observe that the fidelity of initializing a superposition is maximized at $\left|s_{0}\right|$, but the fidelity of initializing an eigenstate is minimized. This is because at $\left|s_{0}\right|$ fluctuations in nuclear field have the greatest effect on the orientation of the eigenstate on the Bloch-sphere. Conversely, exciting a superposition using an optical transition with a finite line-width leads to a jitter in the time at which the exciton is created. This acts to reduce the initial degree of polarization when the coherent oscillations are faster at large $|s|$. Thus the time-averaged degree of polarization displays a maximum in the fidelity of superposition as the FSS is tuned through $\left|s_{0}\right|$ [26]. The width of this variation and the maximum degree of polar- ization is determined by the fluctuations and dephasing processes that control the free-induction decay, not the line-widths of the individual exciton transitions.

The arguments presented here are also relevant to the case of entangled photon pair generation, where decay of the biexciton prepares the exciton in a given superposition [28]. Many publications have stressed the importance of reducing the FSS for a given dot to allow for the emission of entangled photon pairs [5]. This condition is not necessary when temporal filtering can be applied to resolve the coherent oscillations of the exciton [27]. In addition to these considerations we stress here that the only necessary and sufficient condition for entangled photon emission is that the $T_{2}$ decay of the $X$ state not be faster than the radiative lifetime or detector response time, which ever is lower. If this is the case the fidelity of entangled photon pair emission cannot be re-claimed through temporal filtering.

In conclusion, we have shown the effects of nuclear field fluctuations in the semiconductor environment cannot be ignored when considering the evolution of a superposition trapped in a single quantum dot. These fluctuations lead to a reduction in the degree of polarization for the superposition, in a process closely analogous to the freeinduction decay of spins in nuclear magnetic resonance. An interesting avenue for future research would be the combination of our measurements and the use of spinpumping to partially align the nuclear field.

\section{ACKNOWLEDGEMENTS}

This work was partly supported by the EU through the Integrated Project QESSENSE, ITN Spin-optronics and EPSRC.
[1] F. Bloch, Phys. Rev. 70, 460-474 (1946).

[2] P. Neumann et al, Science. 329, 542-544(2010).

[3] A. Morello et al, Nature. 467, 6876914 (2010).

[4] P. Michler et al, Science 290, 2282-2285 (2000).

[5] R. M. Stevenson et al, Nature 439, 179-182 (2006).

[6] T. Flissikowski et al, Phys. Rev. Lett. 86, 3172 (2001)

[7] S. T. Yilmaz, P. Fallahi, and A. Imamoglu, Phys. Rev. Lett. 105, 033601 (2010)

[8] M. Kroutvar et al, Nature. 432, 81-84 (2004)

[9] M. Atature et al, Science. 312, 551-553 (2006)

[10] A. Boyer de la Giroday et al, Phys. Rev. B 73, 033306 (2010).

[11] A. Greilich et al, Science. 313, 341-345 (2006)

[12] T. D. Ladd etal, Phys. Rev. Lett. 105, 107401 (2010)

[13] X. Xu et al, Nature. 459, 1105-1109 (2009)

[14] C. Latta et al, Nature Phys. 5, 758-763 (2009)

[15] A. I. Tartakovskii et al, Phys. Rev. Lett. 98, 026806 (2007)

[16] P. Maletinsky, A. Badolato, and A. Imamoglu, Phys.
Rev. Lett. 99, 056804 (2007).

[17] P. Fallahi, S. T. Yilmaz, and A. Imamoglu, Phys. Rev. Lett. 105, 257802 (2010)

[18] D. Gammon etal, Phys. Rev. Lett. 86, 5176-5179 (2001)

[19] E. A. Chekhovich, A. B. Krysa, M. S. Skolnick, and A. I. Tartakovskii, Phys. Rev. Lett. 106, 027402 (2011)

[20] A. J. Bennett et al, Nature Phys. 100, 177401 (2010).

[21] S. Haroche, J. A. Paisner, and A. L. Schawlow, Phys. Rev. Lett. 30, 948951 (1973)

[22] M. Bayer et al, Phys. Rev. Lett. 82, 1748-1751 (1999).

[23] R. M. Stevenson et al, Phys. Rev. B. 73, 033306 (2006)

[24] M. Bayer et al, Phys. Rev. B 65, 195315 (1999).

[25] B. Eble et al, Phys. Rev. B 74, 081306 (2006).

[26] K. Kowalik et al, Appl. Phys. Lett. 91, 183104 (2007)

[27] R. M. Stevenson et al, Phys. Rev. Lett. 101, 170501 (2008)

[28] R. M. Stevenson et al, arXiv, 1103.2969 (2011). 